\title{
Lecturas y perspectivas de la ética militar ${ }^{1}$
}

\author{
https://doi.org/10.21830/9789585377127.01
}

David Whetham ${ }^{2}$

King's College London

Andrés Eduardo Fernández Osorio ${ }^{3}$

Escuela Militar de Cadetes "General José María Córdova”

Marina Miron ${ }^{4}$

Escuela Superior de Guerra "General Rafael Reyes Prieto"

\section{Resumen}

Este capítulo describe brevemente los diferentes entendimientos y perspectivas de la ética militar presentes en la cotidianidad de los miembros de las fuerzas armadas. Pone de manifiesto la complejidad de los conflictos armados, la volatilidad de sus escenarios y la dificultad en la toma de decisiones para el empleo de la fuerza militar, enfatizando la nece-

1 Este capítulo presenta los resultados colaborativos de dos proyectos de investigación: (1) "Ética militar en entornos complejos de seguridad y defensa: lecturas y aportes desde la experiencia de las Fuerzas Militares de Colombia”, del grupo de investigación Masa Crítica, de la Escuela Superior de Guerra "General Rafael Reyes Prieto", Colombia, categorizado en B por Minciencias y con código de registro COL0123247, y (2) "Mujeres de arma, seguridad y defensa nacional. Un análisis desde sus percepciones", del grupo de investigación en Ciencias Militares, de la Escuela Militar de Cadetes "General José María Córdova”, Colombia, categorizado en B por Minciencias y con código de registro COL0082556. Los puntos de vista pertenecen a los autores y no reflejan necesariamente los de las instituciones participantes. El texto es una adaptación del publicado en Whetham, D. (2017). Challenges to the professional military ethics education landscape. En Making the military moral (pp. 142-159). Routledge.

$2 \mathrm{PhD}$ en Estudios de Guerra del King's College London. Director del Centro de Ética Militar del King's College London, Reino Unido. OrciD: https://orcid.org/0000-0001-8269-0006 - Contacto: david. whetham@kcl.ac.uk

3 Teniente Coronel del Ejército Nacional de Colombia. PhD en Derecho y Ciencia Política de la Universidad de Barcelona, España. Magíster en Economía, Estado y Sociedad: Política y Seguridad del University College, Londres, Reino Unido. Magíster en Relaciones Internacionales de la Higher School of Economics, Moscú, Federación de Rusia. Investigador del grupo de investigación en Ciencias Militares, de la Escuela Militar de Cadetes "General José María Córdova”. Orcid: https://orcid.org/0000-0003-06430258 - Contacto: andres.fernandez@buzonejercito.mil.co

$4 \mathrm{PhD}$ en Estudios Estratégicos de la Universidad de Nueva Gales del Sur, Academia de las Fuerzas Australianas de Defensa. Investigadora del Centro de Ética Militar del King's College London, Reino Unido. Orcid: https://orcid.org/0000-0003-3695-6541 - Contacto: marina.miron@kcl.ac.uk 
sidad de una educación permanente para el personal uniformado, así como la comprensión de las variables que afectan sus decisiones. De igual forma, sirve como preámbulo de la colección "Ética militar y Fuerza Pública en Colombia”.

Palabras clave: Colombia; ética militar; Fuerza Pública; profesionalismo.

\section{Introducción}

La profesión militar, como todas las profesiones, se define y se rige en gran parte por su ética, es decir, por las reglas y los comportamientos por los cuales se conducen sus miembros. Cualquier fuerza militar profesional, en cualquier parte del mundo, se ve a sí misma como distinta de un "simple" grupo de mercenarios o contratistas a largo plazo, y esa identidad propia se basa en más que simplemente ser un servidor reconocido del Estado, autorizado para emplear la violencia como y cuando sea necesario.

Un cierto grado de autonomía sobre cómo se emplea esa violencia y la adherencia estructurada a las leyes, los códigos y las normas aceptadas es parte de esa identidad. El incumplimiento de esas reglas puede ser legalmente incorrecto y, por lo tanto, hacer que el perpetrador esté sujeto a sanciones legales, pero también es probable que se considere institucionalmente incorrecto en el sentido de que se apreciará como poco profesional.

No es de extrañar que esta ética esté tan bien fundada. Las fallas éticas de los militares pueden tener consecuencias terribles para una gran variedad de personas, incluidas las poblaciones civiles locales y los combatientes de ambos lados de un conflicto, para la salud interna de la propia organización militar, para la relación entre el ejército y la sociedad, y para la utilidad estratégica de las fuerzas comprometidas en nombre de su comunidad política.

El tipo de "conflicto discrecional" que actualmente parece ser la norma, donde los intereses nacionales vitales no están obviamente en juego, puede plantear diferentes desafíos éticos y legales para las democracias en comparación con las guerras de supervivencia nacional, donde los problemas generalmente son mucho más complejos y ambiguos en razón a las particularidades cambiantes de la guerra. La gama potencial de problemas que deben abordarse también se amplía debido a los diversos tipos de actividades en las que pueden involucrarse los militares. 
Las operaciones de mantenimiento de la paz o de imposición de la paz y ayuda humanitaria plantean tipos de desafíos muy diferentes a los que se encuentran en la guerra "tradicional" de alta intensidad de Estado a Estado. En ninguna parte esto es más obvio que en la contrainsurgencia, las operaciones antiterroristas o las guerras irregulares. Estas introducen toda una serie de dilemas éticos y legales que se deben explorar y resolver para que las campañas se lleven a cabo de manera adecuada y, en última instancia, se consideren exitosas.

\section{Ética militar y legitimidad}

Una de las preguntas que surgen a menudo es ¿por qué se trata de una cuestión de ética y no simplemente de legalidad? Se supone que cada uno de los militares profesionales de todo el mundo deben cumplir con sus obligaciones internacionales y garantizar que el derecho de los conflictos armados se enseñe y actualice cada año a la totalidad del personal militar en servicio, tanto en tiempo de paz como en tiempo de guerra.

Si esto se hace correctamente, se garantizará la legitimidad en todas las acciones, pero ¿̨no sería redundante la educación en ética militar? Claramente, existe una gran superposición entre las dos áreas de la ética y el derecho. Sin embargo, a veces el entorno operativo actual presenta situaciones en las que puede no estar claro cuál es realmente la situación jurídica. Por ejemplo, la edición de 1990 del Manual de pequeñas guerras de los Estados Unidos establece:

Las pequeñas guerras exigen el tipo más alto de liderazgo dirigido por la inteligencia, iniciativa e ingenio. Las guerras pequeñas se conciben en la incertidumbre, se llevan a cabo a menudo con responsabilidad precaria y autoridad dudosa, bajo órdenes indeterminadas que carecen de instrucciones específicas. (Cuerpo de Infantería de los Estados Unidos, 1990, pp. 1-6)

Incluso cuando el marco de la ley es absolutamente claro y la gama de opciones legalmente permisibles puede identificarse claramente, la ley no es suficiente por sí sola para proporcionar las respuestas reales. Aún es necesario tomar una decisión sobre qué curso de acción se debe seguir. La toma de 
decisiones militar requiere la capacidad de responder preguntas como "¿Sería lícita tal acción en esta situación?”. Pero al mismo tiempo necesita personas que también pregunten: "Este curso de acción es legal, pero ¿es realmente lo correcto?". Por lo tanto, la mejor toma de decisiones se basará en consideraciones tanto éticas como legales si se desea lograr el resultado más apropiado y deseable.

El tipo de cuestiones que surgen en la ética militar a veces no tiene respuestas sencillas, por lo que puede parecer que existe una gran cantidad de desacuerdos sobre aspectos fundamentales, lo cual lleva a la percepción de que "todo es relativo" dadas las circunstancias en las que se toman decisiones en medio de los conflictos armados. En efecto, dado que el escenario operacional generalmente es volátil, incierto, complejo y ambiguo, los miembros de las fuerzas armadas usualmente se enfrentan a tres variables que pueden llegar a poner a prueba su integridad ética y el desenlace de sus acciones.

Una primera variable que condiciona el accionar militar es la escasez e inexactitud de la información disponible para la adopción de cursos de acción. Si bien la evolución de la tecnología ha permitido a lo largo de los años que las unidades militares cuenten con una mayor rapidez en la consecución de datos y su procesamiento, aún se pueden cometer errores no intencionales en el establecimiento de objetivos militares legítimos que impliquen el uso inadecuado de la fuerza.

Una segunda variable es el limitado tiempo disponible para la toma de decisiones y la premura para utilizar la fuerza militar. El llamado ritmo de la batalla y el tempo operacional, definido por el Ejército de los Estados Unidos (2017, pp. 2-7) como la "velocidad relativa y el ritmo de las operaciones militares con el tiempo con respecto al adversario", implican imprimir la rapidez necesaria para aprovechar las oportunidades y debilidades que el enemigo tiene en su maniobra con el objetivo de hacer uso justo de las armas y doblegar su voluntad de lucha.

Una tercera y última variable es la capacidad de afectación y destrucción del armamento militar y sus posibles daños colaterales. Los sistemas de armamento son diseñados para impactar negativamente al adversario y conducirlo a su rendición a través de la disminución de su personal, equipo e instalaciones, 
lo cual a su vez conlleva la pérdida de la iniciativa y la capacidad de maniobra y de mando y control sobre las unidades. No obstante, la utilización de sistemas de armamento, cada vez más precisos, en ocasiones trae desenlaces fatales por daños colaterales no previstos o que emergen durante el empleo de la fuerza.

Dado lo anterior, es importante señalar que los desacuerdos que surgen tienen un enfoque muy limitado en relación con las complejidades propias de las operaciones militares. Aunque líderes militares y académicos en ética militar se sienten atraídos por el análisis de ejemplos complicados, en los cuales no es sencillo analizar la aplicación de las reglas de conducta ética, también se hace necesario estudiar la vida cotidiana de los miembros de la fuerza pública con el fin de comprender los innumerables retos a los que se enfrentan.

Explorar en forma holística todos los aspectos de la cotidianidad de las fuerzas armadas y los dilemas éticos que estos representan permite comprender la multiplicidad de lecturas que pueden tener, así como establecer su evolución y perspectivas. De igual forma, facilita determinar las circunstancias, fortalezas y debilidades en la preparación en ética militar que requieren sus integrantes.

En la gran mayoría de las circunstancias que plantean un dilema ético para los miembros de la fuerza pública hay una decisión correcta para tomar, así como hay cursos de acción incorrectos, los cuales son mucho más fáciles de identificar y evitar si los uniformados han tenido la oportunidad de estudiarlos con anticipación y se les han proporcionado las herramientas para comprender correctamente la situación.

En el reducido número de casos en los que no se puede determinar el curso de acción correcto y se requiere definir cuál es el curso de acción menos perjudicial, también es esencial una adecuada fundamentación profesional que haya fortalecido el entendimiento de los conceptos y principios que rigen la profesión militar y la responsabilidad que tienen sus miembros, sin detrimento de la efectividad militar.

La efectividad militar, definida en forma genérica como la conducción de la guerra en forma exitosa (Reiter \& Stam, 1998), es quizás uno de los temas más frecuentemente discutidos por las fuerzas armadas, los decisores políticos y la sociedad, toda vez que las victorias militares legítimas contribuyen a consolidar la confianza en las instituciones públicas. Sin embargo, diversas perspec- 
tivas sobre el uso y limitaciones en la aplicación de la fuerza, así como en las reglas de enfrentamiento, en ocasiones generan desacuerdos sobre la toma de decisiones y su impacto en la efectividad militar.

Esta es exactamente la razón por la cual es necesario educar, en lugar de capacitar, en esta importante área de la vida profesional militar. La formación, bien realizada, enseña qué hacer en una situación específica. La educación, bien hecha, consiste en equipar a las personas con las herramientas y las habilidades para que puedan entender y hacer lo correcto en cualquier situación, independientemente de si se han capacitado para ello.

Son precisamente las preguntas para las que no hay respuestas en blanco y negro las que deben ser asumidas, pensadas y discutidas por las personas para las que son más pertinentes, los militares, antes de que esas personas se encuentren en situaciones en las que realmente necesitan tomar esas decisiones. La presente colección de tres volúmenes se planteó como una herramienta más en el avance de la fundamentación profesional en ética militar de los miembros de la fuerza pública en Colombia y como justo reconocimiento a la complejidad de los cambiantes escenarios a los cuales se enfrentan diariamente para la defensa de los Derechos Humanos y el Estado social de derecho.

\section{Referencias}

Cuerpo de Infantería de los Estados Unidos. (1990). FMFRP 12-15 Small wars manual. Departamento de la Armada.

Ejército de los Estados Unidos. (2017). ADRP 3-O Operations. Departamento del Ejército.

Reiter, D., \& Stam, A. C. (1998). Democracy and battlefield military effectiveness. Journal of Conflict Resolution, 42(3), 259-277. https://doi.org/10.1177/0022002798042003003

Whetham, D. (2017). Challenges to the professional military ethics education landscape. En Making the military moral (pp. 142-159). Routledge. 